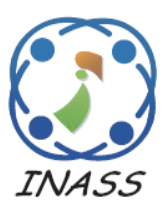

\title{
New Approach for 3D Mesh Retrieval Using Data Envelopment Analysis
}

\author{
Mohcine Bouksim $^{1 *}$ Fatima Rafii Zakani $^{1}$ Khadija Arhid $^{1}$ Mohamed Aboulfatah ${ }^{2}$ Taoufiq Gadi ${ }^{1}$ \\ ${ }^{1}$ Laboratory of Informatics, Imaging, and Modeling of Complex Systems (LIIMSC) \\ Faculty of Sciences and Techniques, Hassan 1st University, Settat, Morocco \\ ${ }^{2}$ Laboratory of Analysis of Systems and Treatment of Information (LASTI) \\ Faculty of Sciences and Techniques, Hassan 1st University, Settat, Morocco \\ * Corresponding author's Email: mbouksim@gmail.com
}

\begin{abstract}
In the last decades, 3D models have known a significant expansion, as we can see them in many fields such as computer vision, biology, augmented reality, engineering, and even medicine. They become more and more popular and accessible than ever. Due to these facts, there is an increasing need for a retrieval system, which can return the most similar 3D objects existing in the database to the query. In the present paper, a new retrieval approach is proposed, based on a multi-criteria method to generate a compact descriptor, which represents a signature for each 3D model. The key idea behind this approach is to try to extract the best out of each criterion (i.e., measure) by extracting a combined score using the Data envelopment analysis method (DEA). The results of the proposed method are very satisfactory and outperform some commonly used retrieval methods which highlight the potential and the performance of our approach.
\end{abstract}

Keywords: 3D object retrieval, 3D shape retrieval, 3D shape matching, Multicriteria method, Data envelopment analysis.

\section{Introduction}

Recently 3D models have known a significant expansion, this is due to the development of GPUs, CPUs, scanning, modeling, and printing tools, in addition to the increasing number of databases available over the World Wide Web. These facts help to make the creation, and the manipulation of 3D models much easier, and less expensive than before. This expansion has made it easy to introduce this kind of model in many fields such as mechanical engineering, biology, medicine, augmented reality, and many other areas will make a use of 3D models soon. The availability of 3D models created the need for a robust retrieval system, which can extract similar objects to a query provided by the user. Conventional search engine based on textual annotation cannot be applied to the 3D field because most used 3D models representations do not contain any semantic information, only geometric information, and even attempting to affect manually textual annotation will be influenced by many factors such as language, culture, perception, and many more. Through the years many researchers studied this problematic, and it turns out that the best results are obtained by creating a signature or a descriptor regrouping local or global geometric, or topologic or any other information extracted from the 3D models (indexing phase); then the search engine will only have to compare between descriptors corresponding to each 3D model in the database (matching phase). Many works have been proposed in this field; we encourage readers to refer to the following works Lara López et al. [1], Yang et al. [2] and Tangelder et al. [3] which provide an extended state of the art and a comparison of many of the existing methods in the literature.

Since generally combining several descriptors or measures usually increase the effectiveness and robustness of the search by the content, we got interested on how can we combine these measures to extract a final compact signature that represents the 3D model. Method called "classic" like the average, 
the sum, the minimum or the maximum, don't give good results since the combined measure can be different -and should be different- to reflect various aspects of the 3D model.

To achieve this task, we propose a new approach for 3D model retrieval, which combines different measures to extract a final descriptor, to be used in the retrieval system. This combination is done using a Multicriteria method, data envelopment analysis (DEA) [4], which generates a final descriptor (performance score), where it combines all the inputs measures. The main advantage of our proposal is that we are not limited to use only one measure as conventional descriptors, but we can use as many as available. Besides the use of DEA makes it possible and easy to combine any criteria or measure even if they are of different types. One other major advantage of the proposed method, is when using measures which are invariant under scaling, translation, and rotation the final generated descriptor by DEA keep the same properties.

The remainder of this paper is organized as follow: Section 2 is reserved to a brief state of the art, followed by section 3 where we will give more details about the proposed retrieval approach, then experiment results to prove the effectiveness of the proposed approach. Finally, a conclusion will end this paper.

\section{Related work}

Content-based 3D model retrieval can be defined as the processes of extracting a signature or a descriptor that represent the 3D object in a unique and compact way, instead of using the whole 3D model which is more difficult to manipulate. This step is done offline for existing 3D object in the database and online for the query model. After the extraction of these signatures, the second step is computing the similarity between the generated descriptor for the query model and each 3D model available in the database. The aim is to extract the most similar object to the query and give results that are as near as possible to the human perception.

Many methods have been proposed in the literature to resolve the need of 3D retrieval system, based on a diversity of approaches. In the remainder of this section, we will present some common and most used methods briefly. Many classifications of shape matching methods can be found in the literature, in this paper we will focus only on two of them which are: features based, and view-based methods. Note that as explained by [3] these categories are not disjoint since we can find many methods that can be classified into more than one category.

\subsection{Feature based methods}

This category gathers all methods that compute geometric or topological features of the 3D model; it means that each 3D model will be represented by its geometrical or topological properties. The shape matching task will be summarized as a comparison of features between each 3D model. The Extended Gaussian Images (EGI) proposed by Horn in 1984 [5], which represent a 3D model using the surface normals by mapping them into a unit sphere (Gaussian sphere). Osada et al. [6] proposed many shape distribution, the most two used are : D2 Histogram, it generates a histogram based on the Euclidean distances between two randomly selected points from the 3D model (Fig. 1), and the A3 Histogram it computes a histogram of the distribution of angle between three points selected randomly (Fig. 1). The distributions proposed by Osada et al. are easy and fast to compute, but the results obtained using them are not very satisfactory. Shape Spectrum Descriptor (SSD) was proposed by Zaharia et al. in 2001 [7] it computes a descriptor based on the shape index introduced by Koenderink et al. [8]. Since the SSD is not defined on flat areas the obtained result can be misleading. Funkhouser et al. [9] proposed the 3D Harmonics, which divides the 3D model into functions defined on concentric spheres and then uses spherical harmonics to remove orientation information Fig 2. A pretreatment step which consists in a pose normalization is needed to use the 3D harmonics, which can impact the final results if not done correctly.

\subsection{View based methods}

The idea behind this kind of method is to represent a 3D model by representative 2D captures since if two 3D models are similar, they should stay similar from all viewing angles. This kind of method has been extensively studied, and we can found many

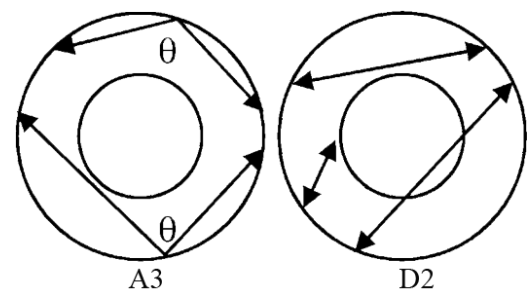

Figure. 1 The representation of both descriptors D2 and A3 [6] 


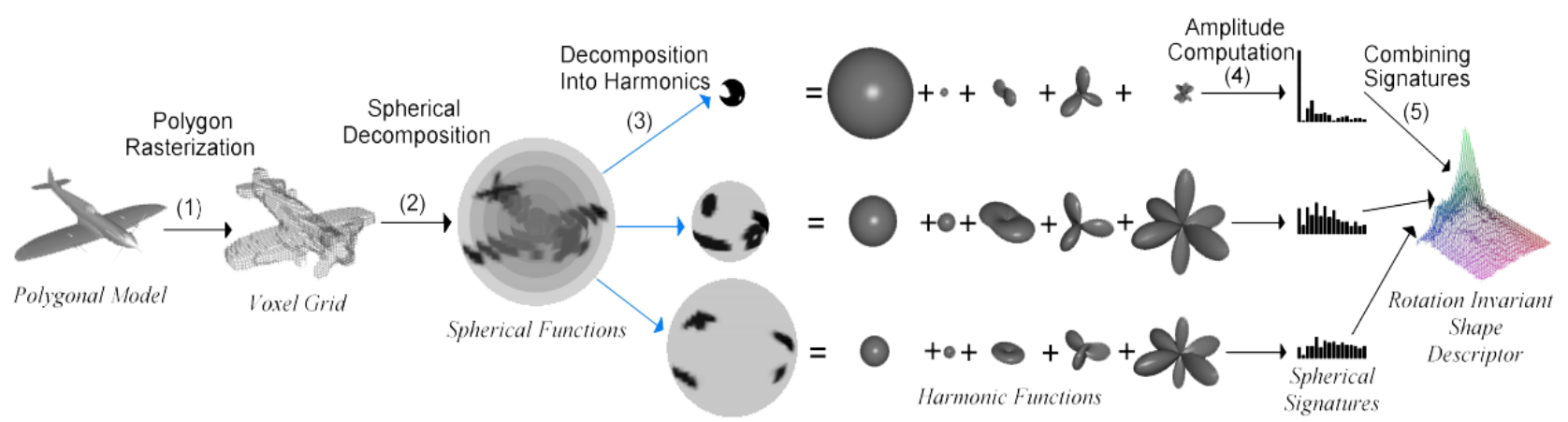

Figure. 2 The representation of the 3D harmonics descriptor [9]

representations like binary images, projection or depth images. The main inconvenient of such methods is that they are not invariant to scaling translation or rotation transformation, so a normalization pretreatment step is needed for these methods. A second inconvenient would be the choice of the number of $2 \mathrm{D}$ projections extracted to represent the 3D model. This number can impact the results heavily since a big number will represent the 3D model in a good way but it will generate a descriptor with big size, and in consequence, the whole retrieval process will be very slow, and vice versa a smaller number of projection will give misleading results.

Ohbuchi et al. [10] proposed a method based on depth images, taken from 42 different viewpoints, for each image a Fourier descriptor [11] is computed, and the set of these 42 vectors is considered as the descriptor for the 3D model. Finally, the similarity is calculated by minimizing the distance between all possible combinations of the two sets of descriptors (query and models from the database). Vranic et al. [12] also proposed a method based on depth images but this time from 6 different viewpoints all perpendicular to the main axis $\mathrm{x}, \mathrm{y}$, and $\mathrm{z}$, once depth images are extracted the authors propose to use discrete Fourier coefficients as a descriptor.

Ouazzani et al. [13] proposed a retrieval method based on binary images AKA Characteristic level images. The main idea behind this method is to take several binary images along each axis; then a clustering algorithm is applied (the authors choose $\mathrm{K}$ mean) to cluster the captured image and take a representative image(the nearest one to the centroid) for each cluster.

Atmosukarto et al. [14] proposed a method for view-based 3D object retrieval. The authors present a new way for selecting salient $2 \mathrm{D}$ views based on learning approach which uses shape characteristics of the $3 \mathrm{D}$ points. The extracted $2 \mathrm{D}$ views are then used to identify the 3D object. Finally, the authors used a similarity measure developed by Chen et al. [15] to compute the similarity between the salient views of the query object and the ones extracted from all the objects in the database.

\section{The proposed retrieval method}

In this section, we will expose our approach for 3D models retrieval. As mentioned before the key idea is to try to compute different measure for the same 3D object and then merge them to extract a final descriptor that combines the advantages of each combined measure. To achieve this task, we choose three criteria to be used dihedral angle, shape index, and the Shape Diameter Function. These three measures are selected for their discriminative power and their invariance under scaling, translation, and rotation. After computing these measures we compute a histogram corresponding to each one of them and finally, we combine these measures using a Multicriteria optimization method. We choose to use data envelopment analysis (DEA) [4] to achieve this task. Finally, we get a final descriptor representing the $3 \mathrm{D}$ object.

\subsection{Selected measures}

Dihedral angle: The dihedral angle is one of the most known measures in the 3D fields, it is defined as the angle between two adjacent planes, in our case it is the angle between two adjacent faces. The dihedral angle between two faces $f_{i}$ and $f_{j}$ is computed as follow:

$$
\theta\left(f_{i}, f_{j}\right)=\arccos \left(\frac{\operatorname{dot}(\vec{u}, \vec{v})}{|\vec{u}||\vec{v}|}\right)
$$

Where $\vec{u}$ and $\vec{v}$ are respectively the normal vector of the face $f_{i}$ and $f_{j} \cdot \overrightarrow{|u|}$ is the norm of the vector $\vec{u}$.

Finally, we affect to each face the average angle between the current face and all its adjacent faces. 
Shape Diameter Function: SDF [16] is described as a scalar function defined on the mesh representing its volume or thickness. It computes a measure of the neighborhood diameter at each point on the 3D mesh. The SDF is computed by taking a cone centered around each point's inward-normal on the 3D mesh; then we send many rays to the other side of the mesh. Finally, we sum of all rays length.

Shape Index: Firstly proposed by Koenderink and van Doorn [8], The shape index is a value that represents the topology of the local surface using the principal curvatures. It is computed using the following formula:

$$
s=\frac{2}{\Pi} \arccos \left(\frac{k_{2}-k_{1}}{k_{2}-k_{1}}\right)
$$

Where $k_{1}$ and $k_{2}$ are the principal curvatures with $k_{2} \geq k_{1}$.

This index is widely used in segmentation and has been already used in 3D mesh retrieval [7].

After computing all these features, we generate a histogram by counting for each measure how many values fall into each of the fixed sized bins, with all the bins having the same size.

\subsection{Data envelopment analysis (DEA)}

Data envelopment analysis (DEA) also known as frontier analysis was firstly proposed by Charnes, Cooper, and Rhodes in 1978 [4], it is a linear programming method, which empirically measures the efficiency of the decision-making units (or DMUs) when this later present multiple inputs and/or outputs. Charnes et al. defined DEA as: "a mathematical programming model applied to observational data, which provides a new method of obtaining empirical estimates of extremal relations such as the production functions and/or efficient production possibility surfaces that are fundamental to modern economics." This technique has been used in many domains such as banks, hotels, hospitals, and university departments.

Through the years many variations and models of the DEA have been proposed [17-18] in the present paper we choose to use the one proposed by Cook \& Kress [19], who propose a new extension of DEA meant to analyze ranked voting data. Following the model proposed by Cook et al. [19] the preference score of each DMU is computed based on a weighted sum of votes with respect to certain weights, the mathematical formula of the voting system is as follow:

$$
\begin{aligned}
& Z_{i}=\text { Maximize } \sum_{j=1}^{k} W_{i} v_{i j} \\
& \text { Subject to }: \sum_{j=1}^{k} W_{i} v_{i j} \leq 1, i=1, \ldots . m \\
& W_{i}-W_{j+1} \geq d(j, \cdot)
\end{aligned}
$$

Where

- $j=1,2, \ldots ., k-1$ and $W_{k} \geq d(j, \epsilon)$

- $W_{j}$ is the weight of the $j^{\text {th }}$ place,

- $v_{i j}$ represents the number of $j^{\text {th }}$ place votes of candidate,

- $d(j, \epsilon)$ is a nonnegative function called the discrimination intensity function.

- $\mathrm{m}$ and $\mathrm{k}$ are respectively the number of candidate and the number of place votes.

After extracting the preference score $Z_{i}$ based on the computed measure, the preference score is considered as a descriptor of each 3D model and will be used in the retrieval process.

\section{Experimental results}

The last section of this paper is reserved to the experimental tests, through 4 sets of tests we will show the effectiveness and the discriminative power of the proposed retrieval approach.

First, and before going throw the experimental test we had to choose which database to use. We can find many over the internet such as Princeton shape benchmark (PSB) [20], Shape Recognition Contest (SHREC) and National Taiwan University database [15]. In our work, we choose to use Princeton's segmentation benchmark database [21], which is a modified version of the Watertight Track of the 2007 SHREC Shape-based Retrieval Contest [22].The choice of the database have been influenced by many criteria, some of them are the number and the diversity of the models. This database contains 380 3D models divided into 19 classes (Human, Cup, Glasses, Airplane, Ant, Chair, Octopus, Table, Teddy, Hand, Plier, Fish, Bird, Armadillo, Bust, Mech, Bearing, Vase, and Fourleg). Another fact is that many models from different categories share the same global form like birds and airplanes, tables and chairs.

The first test is done to choose which distance to use to compare two descriptors or signatures because a good choice of a distance can heavily impact the results of the retrieval system. To achieve this task we compare five different distances (Euclidean distance, 
Table 1. The obtained results for the top 5 nearest neighbor using different distance

\begin{tabular}{|c|c|}
\hline Distance & Score $(\boldsymbol{\%})$ \\
\hline City block & 83.15 \\
\hline Euclidean & 79.84 \\
\hline Chebyshev & 69.31 \\
\hline Cosine & 79.36 \\
\hline EMD & 66.26 \\
\hline
\end{tabular}

Chebyshev, cosine, earth mover's distance and city block) by extracting the k-nearest neighbor for all the possible query (380 in total), we choose $\mathrm{k}$ equal to five. Table 1 summarizes the obtained results.

As we can see from the results, the city block distance gives the best results up to $83 \%$ of correctly retrieved 3D models followed by the Euclidian and the Cosine distances with over $79 \%$ of correctly extracted objects. According to these results in all the remaining experiments, the city block distance will be used to compare signature between models.

The second test is meant to show the utility of the preference score generate by DEA in combining and extracting the best score from the used measures. To do so, we will take two random queries and verify the results using each of the used measures independently along with D2, SSD and use the combined score generated by the DEA.

The figures below (Fig. 3 and Fig. 4) show the results (The query model is the one in the upper middle, and the most similar objects are ordered from left to right and top to bottom). As we can see from the results for the first query (glasses), the proposed method succeed to return 15 out of 20 which is the best score from all the used method for this test followed by SDF and SSD. The second query which represents a table our method got the best score up to 18 out of 20 correctly retrieved objects, followed by SSD and shape index. This test shows the effectiveness of the proposed method compared with other retrieval method but also demonstrate that the obtained score using DEA which combines three measures is better than using each measure individually.

The third test will present some sample results obtained using SSD, D2 and our proposed approach; Figure 5 shows the obtained results, the models in the leftmost column show the query 3D models (chosen randomly one model per class), while the columns on the right show the closest matches among the used database. From the obtained results we can easily see that our approach succeed to provide excellent results. For example, a query representing a cup, a fish, an armadillo, a chair or a teddy bear our retrieval system succeed to give six out of six correct 3D objects, SSD didn't succeed to do the same for the cup object and D2 with the worst result got four out of six correctly retrieved object in the best case . For other queries like human or bust the proposed approach returns five out of six correct objects which are once again way better than what D2 and SSD achieve.

The fourth test is done to investigate the discriminative power of the proposed method between classes of 3D objects. To do so, we compute a similarity matrix between all pairs of $3 \mathrm{D}$ objects available in the used database; the results are exposed in Fig. 6. In this figure, each row and column represent the similarity between each 3D model and the whole database, which implies that the matrix is symmetric and square. The matrix can be divided into 19X19 blocks each block represents a different class of 3D objects (containing $203 \mathrm{D}$ objects). A robust retrieval method should have darker color in the diagonal's block which means higher similarity between objects within the same class, which is almost the case for the proposed method as we can see from the similarity matrix most of the $3 \mathrm{D}$ objects

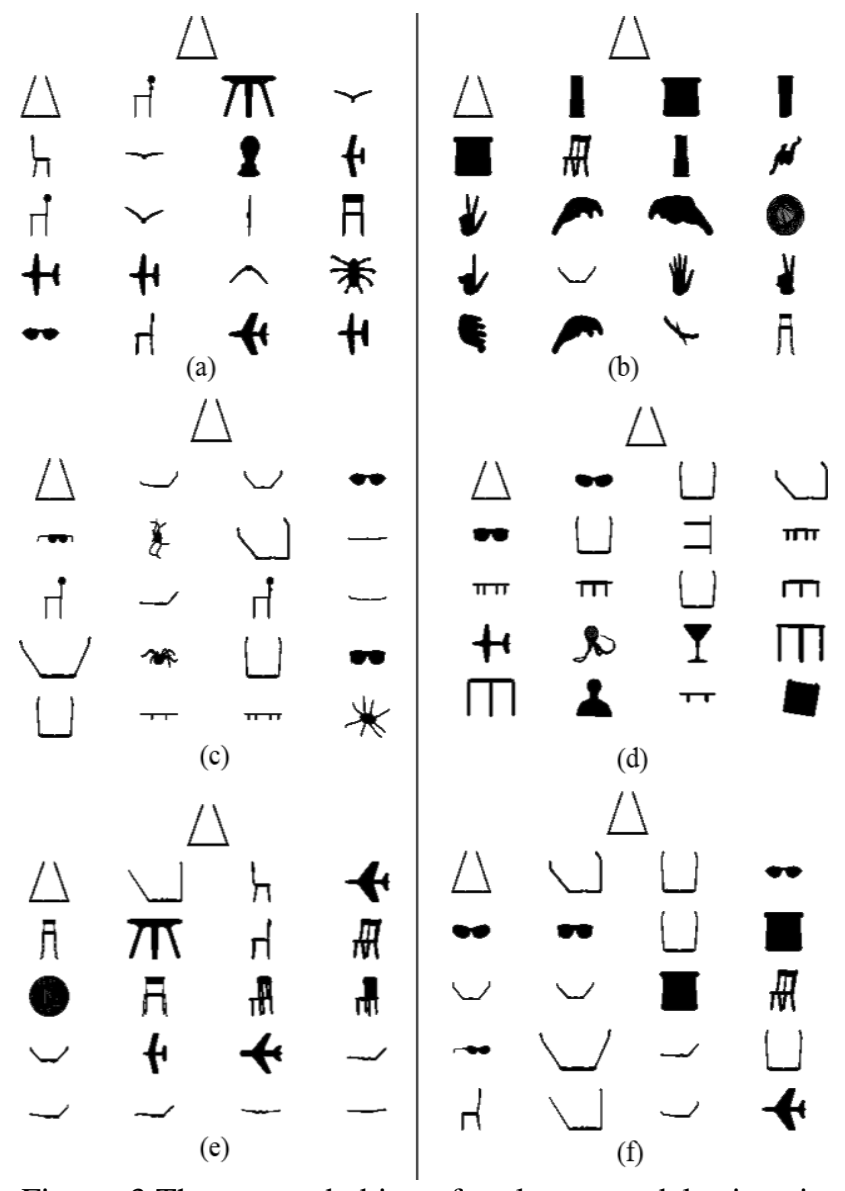

Figure. 3 The returned objects for glasses model using six methods: (a) dihedral angle, (b) shape index, (c) shape diameter fiction, (d) D2, (e) SSD, (f) the proposed method. 


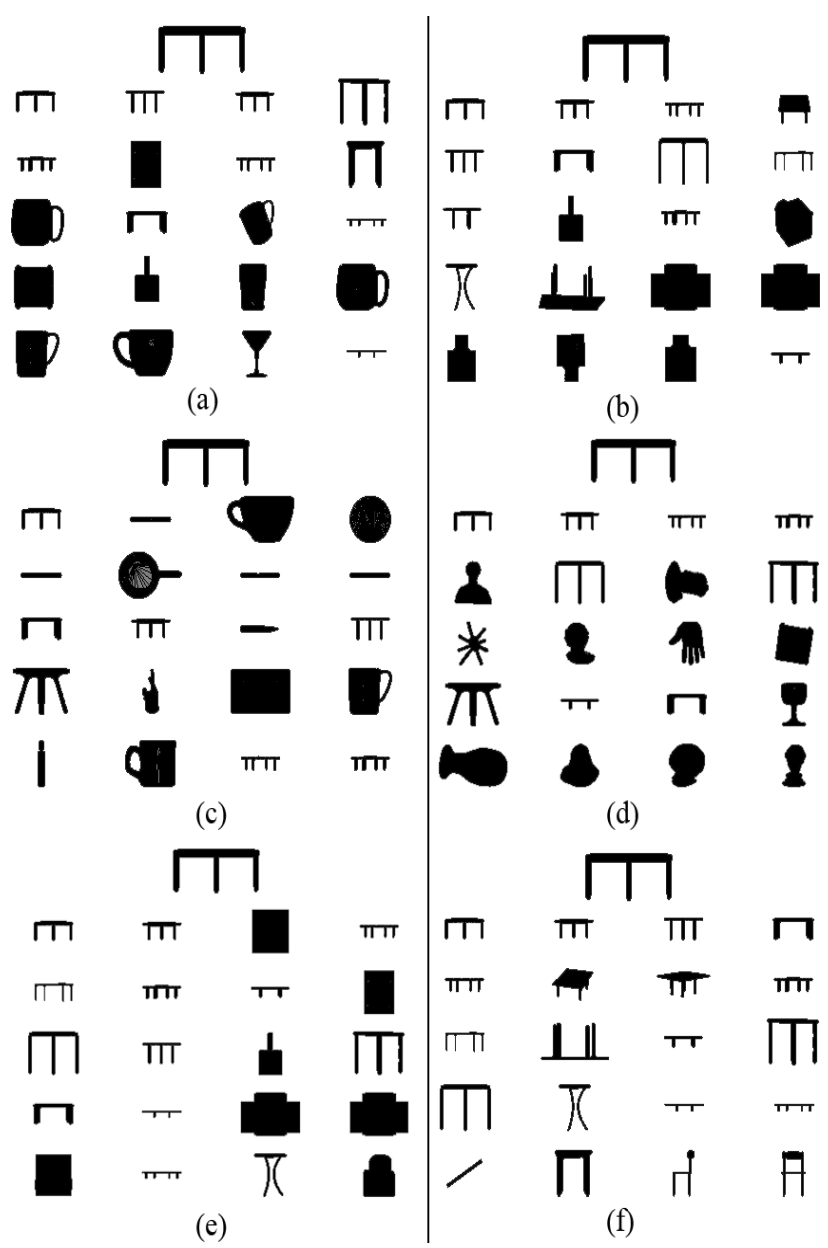

Figure. 4 The returned objects for a table model using six methods: (a) dihedral angle, (b) shape index, (c) shape diameter fiction, (d) D2, (e) SSD, (f) the proposed method.

were assigned to the correct class.

We can also observe from the figure that for some models they got higher similarity with models in different classes like airplane and bird, ant and octopus or table and chair, even if it is not correct from a semantic viewpoint it is correct from a geometric viewpoint since these classes share almost the same general geometric aspect. In the other hand, SSD and D2 didn't succeed to detect the similarity between all classes of 3D models even if some classes were correctly retrieved, especially by SSD like Teddy, chair, or table. Other classes were badly represented for example both methods detect higher similarity between (human and fourleg), (airplane and glasses), (airplane and plier) or between (fish and human), which is not correct from both semantic or geometric view point.

As a final experiment, we will compute the recall and precision diagram, which is considered as a traditional and a common test to evaluate the effectiveness of any retrieval system. The recall measures the relevant results retrieved by the system over the total relevant in the database, and the precision measures the relevant results among the retrieved instances. They are computed using the formula:

$$
\begin{aligned}
& \text { Recall }=\frac{\text { relevant correctly retrieved }}{\text { all relevant }} \\
& \text { Precision }=\frac{\text { relevant correctly retrieved }}{\text { all retrieved }}
\end{aligned}
$$

To make a meaningful comparison we choose to compare the obtained results with those obtained from the following methods: Extended Gaussian Images (EGI) [5], two distribution from Osada et al. work D2, A3 [6], Shape Spectrum Descriptor (SSD) [7] and 3D Harmonics [9].

Figure 7 represents the precision-recall curves obtained for the proposed method along with those cited before. From the Fig. 7, we can observe that the proposed method outperforms all the other methods especially D2, A3, and the Extended Gaussian Images (EGI). After all these experiments we can deduce that the proposed method performs very well and gives very satisfactory results and can retrieve very good number of correct object based on a query model, compared with other well-known retrieval methods.

\section{Conclusion}

In this paper, we present a new method for indexation and retrieving 3D models. The proposed approach combines many measures to extract one last descriptor using a multicriteria method DEA. The obtained results are very satisfactory and outperform many well-known methods in term of discriminative power and effectiveness. The proposed approach can be used to combine any measures, without any specific order or any pretreatment, for example, we can combine local and global geometric features to extract a representative score for the whole $3 \mathrm{D}$ object using DEA method. For future works, we plan to investigate other measures to be combined and also test another approach to merge this measures. We are also testing our approach on a view based approach (2D projections).

\section{Acknowledgments}

The authors would like to thank Xiaobai Chen et al. for providing the database from "Benchmark for 3D Mesh Segmentation" online on their website 
(http://segeval.cs.princeton.edu/).We would also like to thank Michael Kazhdan for making available the executable code for the Spherical Harmonic. Finally, we thank the anonymous reviewers for their constructive and valuable suggestions and comments to improve the present paper.

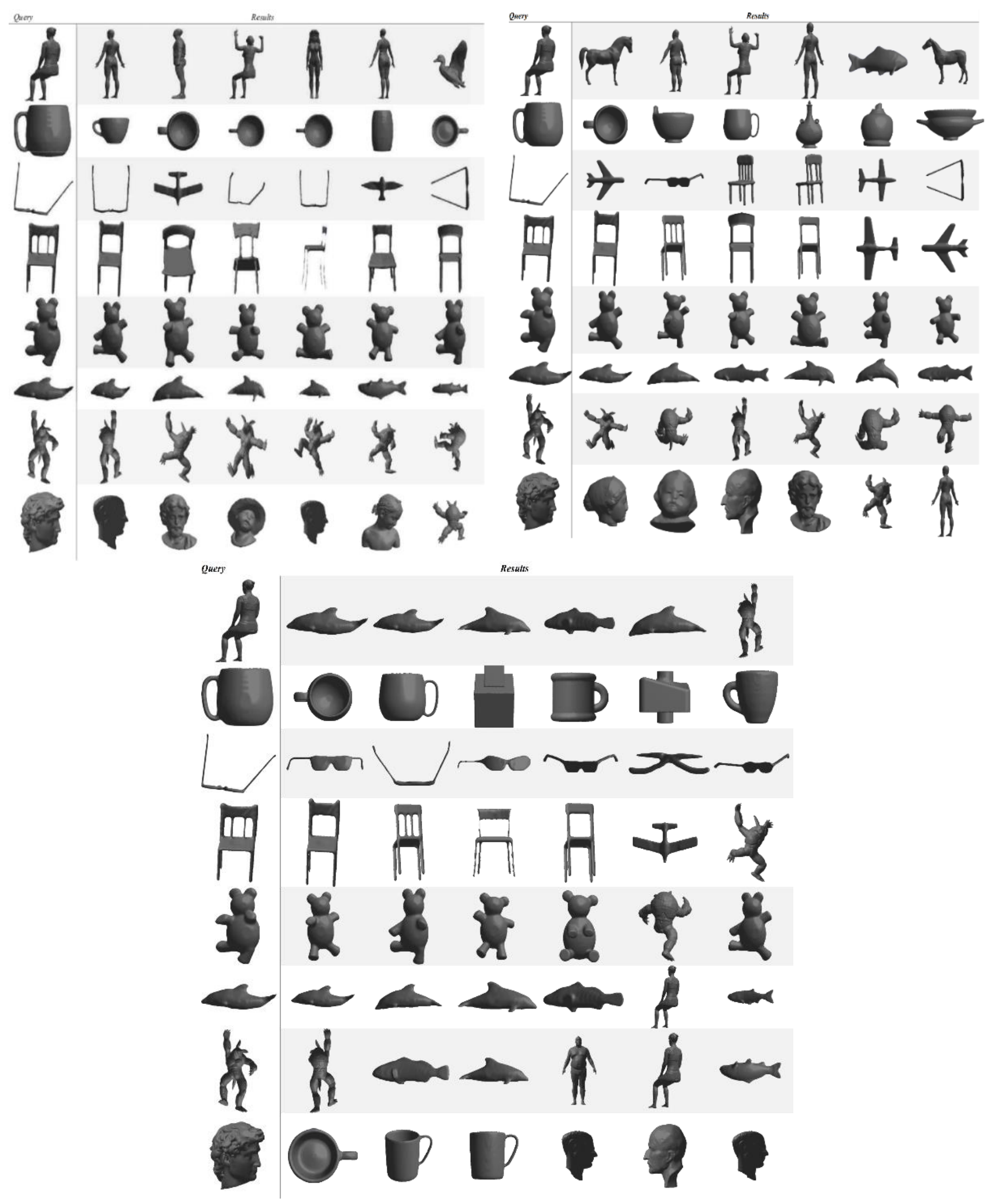

Figure. 5 Top 6 retrieved 3D models using the proposed approach (top left), Shape Spectrum Descriptor (SSD) (top right) and D2 distribution (bottom center) 

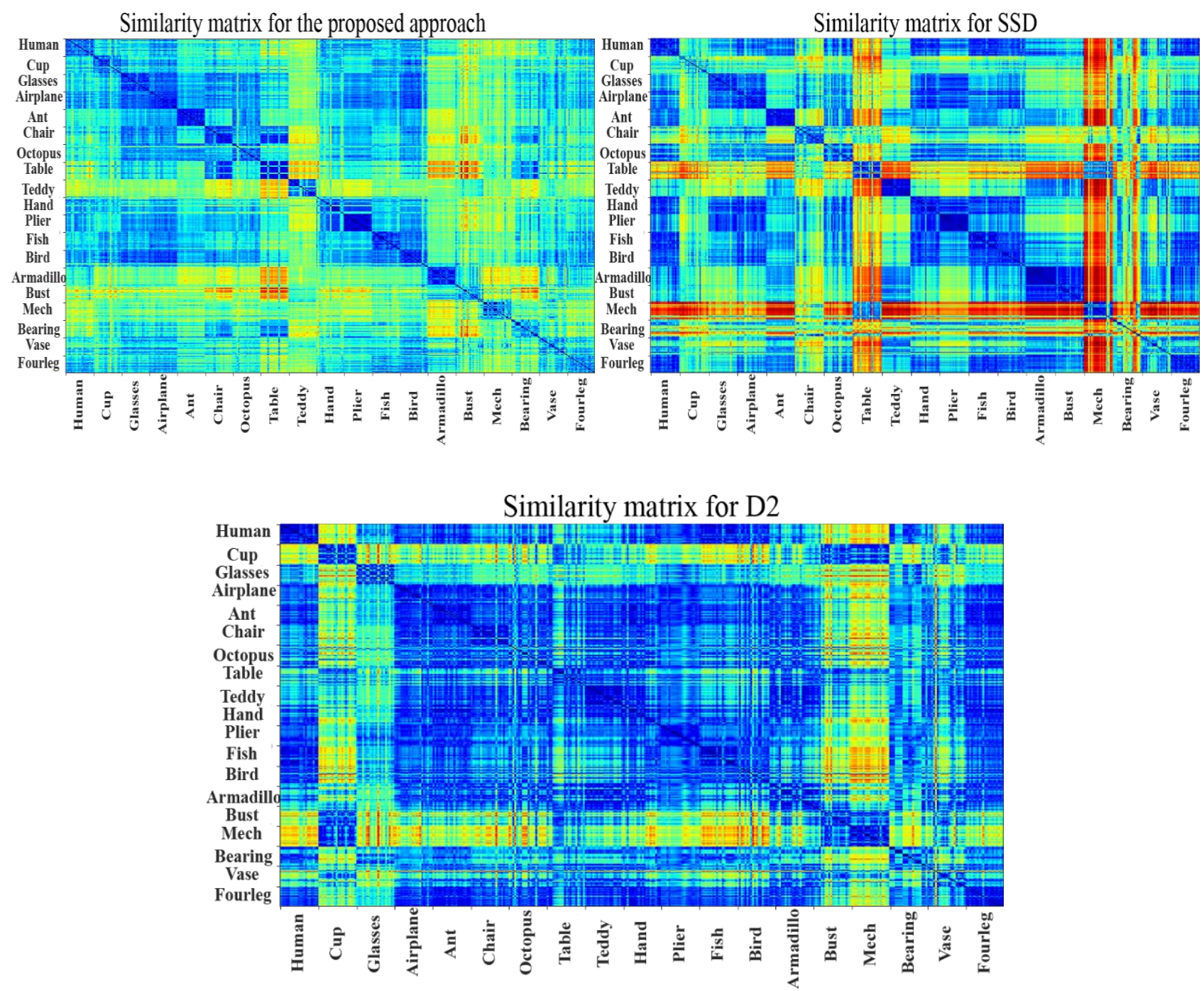

Figure. 6 Similarity matrix for the used database (380 objects divided into 19 classes) using the proposed approach, D2 and SSD

\section{References}

[1] G. Lara López, A. Peña Pérez Negrón, A. De Antonio Jiménez, J. Ramírez Rodríguez, and R. Imbert Paredes, "Comparative analysis of shape descriptors for 3D objects", Multimedia Tools and Applications, Vol.76, No.5, pp.6993-7040, 2017.

[2] Y. Yang, H. Lin, and Y. Zhang, "Content-Based 3-D Model Retrieval: A Survey", IEEE Transactions on Systems, Man and Cybernetics, Part C (Applications and Reviews), Vol.37, No.6, pp.1081-1098, 2007.

[3] J. W. H. Tangelder and R. C. Veltkamp, "A survey of content based 3D shape retrieval methods", Multimedia Tools and Applications, Vol.39, No.3, pp.441-471, 2008.

[4] A. Charnes, W. W. Cooper, and E. Rhodes,
"Measuring the efficiency of decision making units", European Journal of Operational Research, Vol.2, No.6, pp.429-444, 1978.

[5] B. K. P. Horn, "Extended Gaussian Images", Proceedings of the IEEE, Vol.72, No. 12, pp.1671-1686, 1984.

[6] R. Osada, T. Funkhouser, B. Chazelle, and D. Dobkin, "Shape distributions", $A C M$ Transactions on Graphics, Vol.21, No.4, pp 807-832, 2002.

[7] T. Zaharia and F. J. Preteux, "3D-shape-based retrieval within the MPEG-7 framework", In: Proc. Nonlinear Image Processing and Pattern Analysis XII, San Jose, CA, USA, pp.133-145, 2001.

[8] J. J. Koenderink and A. J. van Doorn, "Surface shape and curvature scales", Image and Vision Computing, Vol.10, No.9, pp.557-564, 1992. 


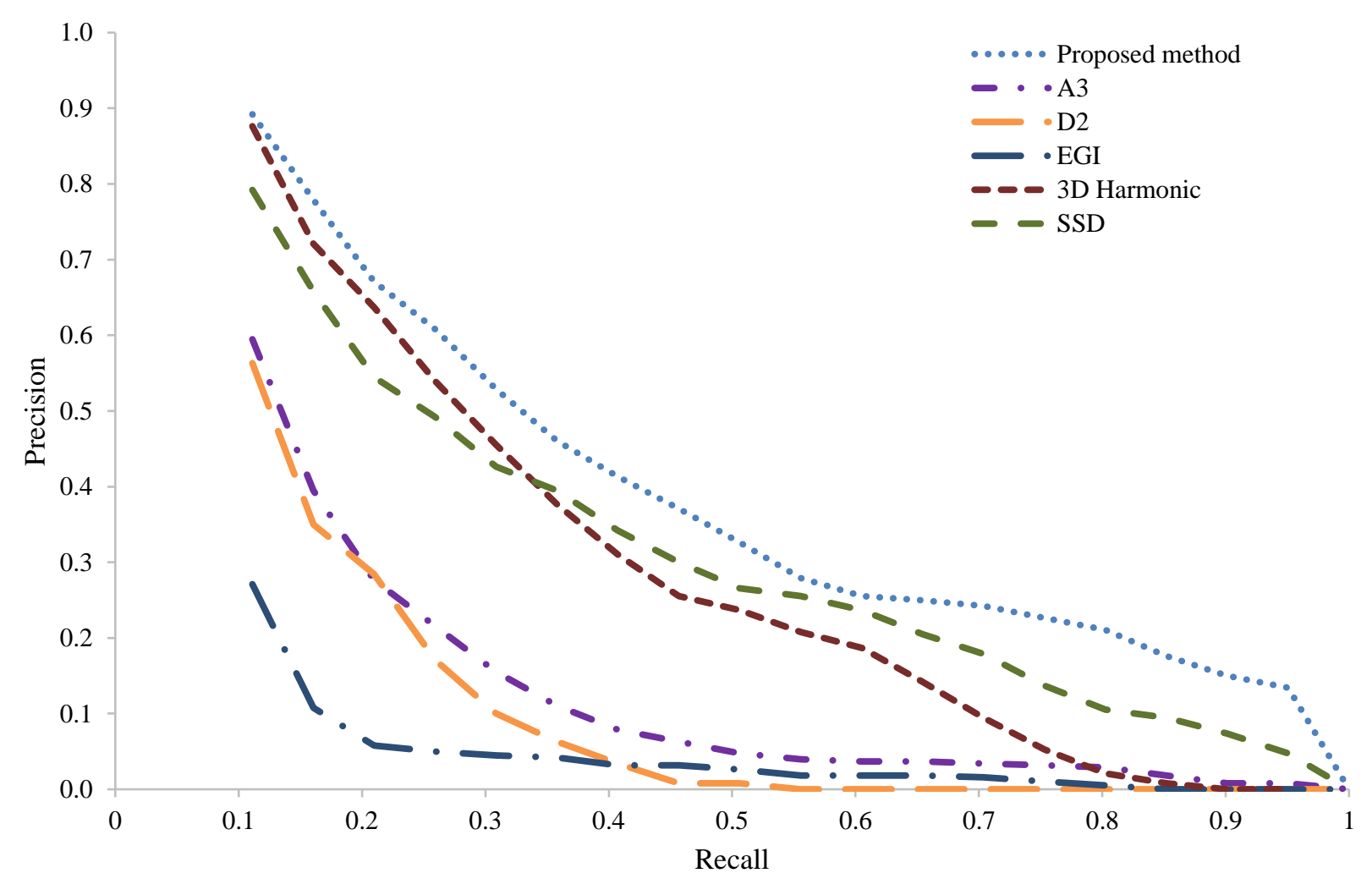

Figure. 7 Precision-Recall graph using five different descriptors and the proposed one

[9] T. Funkhouser, P. Min, M. Kazhdan, J. Chen, A. Halderman, D. Dobkin, and D. Jacobs, "A search engine for 3D models", $A C M$ Transactions on Graphics, Vol.22, No.1, pp.83105, 2003.

[10]R. Ohbuchi, M. Nakazawa, and T. Takei, "Retrieving 3D shapes based on their appearance", In: Proc. of the 5th ACM SIGMM international workshop on Multimedia information retrieval - MIR '03, New York, USA, pp.39-45, 2003.03.

[11]D. Zhang and G. Lu, "Shape based image retrieval using generic fourier descriptor", Signal Processing: Image Communication, Vol.17, No.10, pp.825-848, 2002.

[12]D. V. Vranic, “3D Model Retrieval”, Ph.D. thesis, University of Leipzig, 2004.

[13]I. Ouazzani Taybi, R. Alaoui, F. Rafii Zakani, K. Arhid, M. Bouksim, and T. Gadi, "A novel efficient 3D object retrieval method based on representative slices", In: Proc. International Conference on Multimedia Computing and Systems, Tanger, Morocco, pp. 639-644, 2016.

[14]I. Atmosukarto and L. G. Shapiro, "3D object retrieval using salient views", International Journal of Multimedia Information Retrieval, Vol.2, No.2, pp.103-115, 2013.

[15]D.-Y. Chen, X.-P. Tian, Y.-T. Shen, and M.
Ouhyoung, "On Visual Similarity Based 3D Model Retrieval", Computer Graphics Forum, Vol.22, No.3, pp.223-232, 2003.

[16]L. Shapira, A. Shamir, and D. Cohen-Or, "Consistent mesh partitioning and skeletonisation using the shape diameter function", The Visual Computer, Vol.24, No.4, pp.249-259, 2008.

[17]J. S. Liu, L. Y. Y. Lu, W.-M. Lu, and B. J. Y. Lin, "Data envelopment analysis 1978-2010: A citation-based literature survey", Omega, Vol.41, No.1, pp.3-15, 2013.

[18]C. Sun and X. Gui, "Data Envelopment Analysis: Surveys", In: Proc. of the International Conference on Management and Service Science, Wuhan, China, pp.1-4, 2011.

[19]W. D. Cook and M. Kress, "A Data Envelopment Model for Aggregating Preference Rankings", Management Science, Vol.36, No.11, pp. 1302-1310, 1990.

[20]P. Shilane, P. Min, M. Kazhdan, and T. Funkhouser, "The Princeton Shape Benchmark", In: Proc. of Shape Modeling International SMI, Genova, Italy, pp.167-178, 2004.

[21] X. Chen, A. Golovinskiy, and T. Funkhouser, "A benchmark for 3D mesh segmentation", ACM Transactions on Graphics, Vol.28, No. 3, 
p.1, 2009.

[22]D. Giorgi, S. Biasotti, and L. Paraboschi, "Shape Retrieval Contest 2007: Watertight
Models Track", Technical Report UU-CS2007-015, pp.5-10, 2007. 